\title{
SELECTORS FOR BOREL SETS WITH LARGE SECTIONS
}

\author{
ASHOK MAITRA
}

\begin{abstract}
We prove a result asserting the existence of a Borel selector for a Borcl set in the product of two Polish spaces. This subsumes a number of results about Borel selectors for Borel sets having large sections.
\end{abstract}

1. Introduction. The principal selection theorems for Borel sets with large sections can be stated as follows. If $X$ is analytic, $Y$ a Polish space, $B$ a Borel subset of $X \times Y$ such that every vertical section $B_{x}$ is of positive measure or nonmeager, then $B$ admits a Borel measurable selector, i.e., there exists a Borel measurable function $f$ : $X \rightarrow Y$ such that $(x, f(x)) \in B$ for each $x \in X$. The measure result was proved by Blackwell and Ryll-Nardzewski [1], while the category result has been observed by several authors (see, for example, [5 and 6]). A selection theorem of Burgess [2], when specialized to the situation above, extends the category result and subsumes a result of Srivastava [8] concerning Borel selectors for the case when the sections $B_{x}$ are $G_{\delta}$ sets (see also [4] in this connection). A precise version of Burgess' result will be given in $\S 4$.

The aim of the present article is to show that the results described above are really instances of a single theorem. Moreover, the proof we give here is elementary in nature, using nothing more than the well-known characterization of a Borel subset of a Polish space as a one-one, continuous, bimeasurable image of a closed subset of the space of irrationals (see, for instance, [3]). In particular, the separation principle for analytic sets is avoided. Lastly, the proof works for $X$, an arbitrary separable metric space (Srivatsa [7] observed that the results mentioned in the previous paragraph hold for separable metric $X$, not just for analytic $X$ ).

The situation obtaining for Borel sets with large sections should be contrasted with that for Borel sets with small sections, i.e., when all vertical sections are countable. or compact or $\sigma$-compact. It is known that such Borel sets admit Borel selectors when the horizontal axis $X$ is analytic [5]. The proofs of these results, however, involve in an essential manner the separation principle for analytic sets (indeed, the separation principle for a sequence of analytic sets). Furthermore, the results are not true for arbitrary separable metric $X$. (I am indebted to J. P. Burgess for the last remark.)

Received by the editors February 9, 1983.

1980 Mathematics Subject Classification. Primary 54C65, 28B20, 28A05, 54H05; Secondary 03E15, $04 \mathrm{~A} 15$.

Key words and phrases. Borel selectors, Borel sets, measure, category. 
The main result of this paper is as follows.

Theorem. Let $X$ be a separable metric space and $Y$ a Polish space. For each $x \in X$, let $Y_{x}$ be a $\sigma$-ideal of subsets of the Borel $\sigma$-field of $Y$ such that for each Borel set $C$ in $X \times Y$, the set $\left\{x \in X: C_{x} \notin \mathscr{q}_{x}\right\}$ is Borel in $X$, where $C_{x}=\{y \in Y:(x, y) \in C\}$.

Let $B$ be a Borel set in $X \times Y$ such that $B_{x} \notin \mathcal{F}_{x}$ for each $x \in X$. Then there is a Borel measurable function $f: X \rightarrow Y$ such that $(x, f(x)) \in B$ for each $x \in X$.

The proof of the theorem will be given in $\S 3$. $\$ 2$ explains the notation to be used. $\$ 4$ will discuss special cases of our result.

2. Notation. We denote the set of natural numbers by $\omega$. The letters $k, m, n$, with or without primes, will stand for natural numbers. The set of finite sequences of natural numbers is denoted by $\omega^{<\omega}$. Elements of $\omega^{<\omega}$ will be denoted by $s, t$, with or without primes. The letter $e$ will denote the empty sequence in $\omega^{-\omega}$. If $s \in \omega^{-\omega}$, $\operatorname{lh}(s)$ denotes the length of $s$. If $s \in \omega^{<\omega}$ and $m \in \omega, s m$ denotes the catenation of the sequence $s$ followed by the sequence $\langle m\rangle$.

The set of infinite sequences of natural numbers will be denoted by $\omega^{\omega}$. Elements of $\omega^{\omega}$ will be denoted by $\alpha, \beta$. For $\alpha \in \omega^{\omega}$ and $n \in \omega, \alpha(n)$ stands for the $n$th coordinate of $\alpha$ and $\alpha \uparrow n$ will denote the finite sequence $\langle\alpha(0), \alpha(1), \ldots, \alpha(n-1)\rangle$. For $s \in \omega^{<\omega}$ and $\alpha \in \omega^{\omega}$, write $s \subseteq \alpha$ if $\alpha \uparrow \operatorname{lh}(s)=s$. We denote the set $\left\{\alpha \in \omega^{\omega}\right.$ : $s \subseteq \alpha\}$ by $N(s)$ for $s \in \omega^{<\omega}$. The sets $N(s), s \in \omega^{<\omega}$, form a base for a topology on $\omega^{\omega}$. Endowed with this topology, $\omega^{\omega}$ becomes a Polish space which is homeomorphic to the space of irrationals.

3. Proof of theorem. Fix a complete metric $d$ on $Y$ such that $d-\operatorname{diameter}(Y)<1$. Choose a system $\left\{V(s): s<\omega^{<\omega}\right\}$ of subsets of $Y$ satisfying:

(i) $V(e)=Y$.

(ii) $V(s)$ is nonempty and open in $Y$.

(iii) $V(s)=\cup_{m \geqslant 0} V(s m)$.

(iv) $\operatorname{cl}(V(s m)) \subseteq V(s)$, where cl denotes closure in $Y$.

(v) $d$ - diameter $(V(s))<2^{-\ln (s)}$.

Let $\tilde{X}$ be a completion of $X$, so $\tilde{X}$ is a Polish space. Since $B$ is Borel in $X \times Y$, we can find a Borel set $\tilde{B}$ in $\tilde{X} \times Y$ such that $B=\tilde{B} \cap(X \times Y)$. Fix a closed subset $D$ of $\omega^{\omega}$ and a one-one, continuous function $g$ on $D$ onto $\tilde{B}$ such that $g$ takes Borel subsets of $D$ to Borel sets in $\tilde{X} \times Y$.

Next we define a system $\left\{T(s, t): s, t \in \omega^{<\omega} \& \operatorname{lh}(s)=\operatorname{lh}(t)\right\}$ of subsets of $X$ satisfying:

(a) $T(s, t)$ is a Borel set in $X$,

(b) $T(e, e)=X$,

(c) $T(s, t) \cap T\left(s^{\prime}, t^{\prime}\right)=\varnothing$ if $(s, t) \neq\left(s^{\prime}, t^{\prime}\right) \& \operatorname{lh}(s)=\operatorname{lh}\left(s^{\prime}\right)$,

(d) $T(s, t)=\cup_{m \geqslant 0} \cup_{n \geqslant 0} T(s m, t n)$,

(e) $T(s, t) \subseteq(g(D \cap N(s)) \cap(X \times Y))^{* V(t)}$,

where $(g(D \cap N(s)) \cap(X \times Y))^{* V(t)}=\left\{x \in X:(g(D \cap N(s)) \cap(X \times V(t)))_{x} \notin\right.$ $\left.\mathscr{q}_{x}\right\}$. The definition of $T(s, t)$ is by induction on $\ln (s)$. Start the definition by setting $T(e, e)=X$. Assume that $T\left(s^{\prime}, t^{\prime}\right)$ has been defined for all $s^{\prime}, t^{1} \in \omega^{<\omega}$ such that 
$\operatorname{lh}\left(s^{\prime}\right)=\operatorname{lh}\left(t^{\prime}\right) \leqslant k$. Fix $s, t \in \omega^{<\omega}$ such that $\operatorname{lh}(s)=\operatorname{lh}(t)=k$. Set

$$
T^{\prime}(m, n)=(g(D \cap N(s m)) \cap(X \times Y))^{* V(t n)} \cap T(s, t), \quad m, n \in \omega .
$$

Since $g(D \cap N(s m))$ is Borel in $\tilde{X} \times Y$, the set $g(D \cap N(s m)) \cap(X \times V(t n))$ is Borel in $X \times Y$. So, by hypothesis, $(g(D \cap N(s m)) \cap(X \times Y))^{* V(t n)}$ is Borel in $X$, hence $T^{\prime}(m, n)$ is Borel in $X$. We claim that $T(s, t)=\cup_{m \geqslant 0} \cup_{n \geqslant 0} T^{\prime}(m, n)$. To see this, let $x \in T(s, t)$. Note that

$$
(g(D \cap N(s)))_{x} \cap V(t)=\bigcup_{m \geqslant 0} \bigcup_{n \geq 0}\left[(g(D \cap N(s m)))_{x} \cap V(t n)\right] .
$$

Since $x \in T(s, t)$, the induction hypothesis and clause (e) above imply that

$$
(g(D \cap N(s)))_{x} \cap V(t) \notin \mathscr{q}_{x} .
$$

Since $\mathscr{q}_{x}$ is a $\sigma$-ideal, it follows that there exist $m, n \in \omega$ such that $(g(D \cap N(s m)))_{x}$ $\cap V(t n) \notin \mathscr{G}_{x}$, so $x \in T^{\prime}(m, n)$. Next, disjointify the sets $T^{\prime}(m, n)$ : get Borel sets $T^{\prime \prime}(m, n)$ in $X$ such that $T^{\prime \prime}(m, n) \subseteq T^{\prime}(m, n), T^{\prime \prime}(m, n) \cap T^{\prime \prime}\left(m^{\prime}, n^{\prime}\right)=\varnothing$ if $(m, n) \neq\left(m^{\prime}, n^{\prime}\right)$, and $\cup_{m \geqslant 0} \cup_{n \geqslant 0} T^{\prime \prime}(m, n)=\cup_{m \geqslant 0} \cup_{n \geqslant 0} T^{\prime}(m, n)$. Finally, set $T(s m, t n)=T^{\prime \prime}(m, n)$. The sets so obtained satisfy clauses (a)-(e).

Define

$$
G=\bigcap_{k \geqslant 0} \bigcup[T(s, t) \times \operatorname{cl}(V(t))],
$$

where the inner union is over all ordered pairs $(s, t) \in \omega^{<\omega} \times \omega^{<\omega}$ such that $\ln (s)=\ln (t)=k$.

We make two observations about $G$ : (1) $G_{x}$ is a singleton for each $x \in X$, and (2) $G \subseteq B$. To see (1), let $x \in X$. Then there is a unique $(\alpha, \beta) \in \omega^{\omega} \times \omega^{\omega}$ such that $x \in T(\alpha|k, \beta| k)$ for all $k \geqslant 0$. This follows from clauses (b)-(d) in the definition of the system $\{T(s, t)\}$. So $G_{x}=\bigcap_{k \geqslant 0} \operatorname{cl}(V(\beta \uparrow k))$, which is a singleton set by (iv), (v) and the fact that $Y$ is Polish. For $(2)$, let $(x, y) \in G$. Find $(\alpha, \beta) \in \omega^{\omega} \times \omega^{\omega}$ such that $x \in T(\alpha \uparrow k, \beta \uparrow k)$ for all $k \geqslant 0$. Hence $\{y\}=\bigcap_{k \geqslant 0} \operatorname{cl}(V(\beta\lceil k))$. Since $x \in$ $T(\alpha \uparrow k, \beta \uparrow k)$ for each $k$, it follows by (e) that $(g(D \cap N(\alpha \nmid k)))_{x} \cap V(\beta \backslash k) \neq \varnothing$ for each $k \geqslant 0$. Consequently, $y \in \bigcap_{k \geqslant 0}(\operatorname{cl}(g(D \cap N(\alpha \mid k))))_{x}$, so $(x, y) \in$ $\cap_{k \geqslant 0} \operatorname{cl}(g(D \cap N(\alpha \uparrow k)))=\{g(\alpha)\}$, using continuity of $g$ and closedness of $D$. Consequently, $(x, y) \in B$.

Let $f: X \rightarrow Y$ be the function whose graph is $G$. Since $G \subseteq B$, it follows that $(x, f(x)) \in B$ for every $x \in x$. To see that $f$ is Borel measurable, observe that for any open set $W$ in $Y$,

$$
f^{-1}(W)=\cup\{T(s, t): \ln (s)=\ln (t) \& \operatorname{cl}(V(t)) \subseteq W\},
$$

so that $f^{-1}(W)$ is Borel in $X$. This completes the proof.

4. Special cases. In this final section, we deduce the results mentioned in the Introduction from our result.

Let $X$ be a separable metric space, $Y$ a Polish space and $B$ a Borel set in $X \times Y$.

$1^{\circ}$. To consider the measure result first, let $Q(x, E), x \in X, E$ a Borel set in $Y$, be a Borel measurable transition function such that $Q\left(x, B_{x}\right)>0$ for each $x \in X$. In our theorem, we take $\mathscr{g}_{x}=\{E \subseteq Y$ : $E$ is Borel $\& Q(x, E)=0\}, x \in X$. It is a 
standard fact of measure theory that the $\sigma$-ideals $g_{x}, x \in X$, satisfy the condition of our theorem, so that the Blackwell-Ryll-Nardzewski result falls out of ours.

$2^{\circ}$. For the category case, assume that $B_{x}$ is nonmeager for each $x \in X$. We take $g_{x}=\{E \subseteq Y: E$ is Borel and meager $\}, x \in X$. That the $g_{x}, x \in X$, satisfy the condition of our theorem is a result of P. S. Novikov and, independently, of Vaught [9]. The category result is then an immediate consequence of our result.

$3^{\circ}$. In Burgess' result, the assumptions on the Borel set $B$ are as follows: (a) the multifunction $x \rightarrow B_{x}$ is Borel measurable, i.e., the set $\left\{x \in X: B_{x} \cap V \neq \varnothing\right\}$ is Borel in $X$ for each open set $V$ in $Y$, and (b) $B_{x}$ is nonmeager in $\operatorname{cl}\left(B_{x}\right)$ for each $x \in X$. We set

$$
\mathscr{y}_{x}=\left\{E \subseteq \operatorname{cl}\left(B_{x}\right): E \text { is Borel and meager in } \operatorname{cl}\left(B_{x}\right)\right\}, \quad x \in X .
$$

A very easy modification of Vaught's proof of the fact mentioned in $2^{\circ}$ now shows that the $\sigma$-ideals $g_{x}, x \in X$, satisfy the condition of our theorem. Our theorem then implies that $B$ admits a Borel measurable selector, which is Burgess' result.

Srivatsa [7] has an ingenious way of deducing Burgess' result from the category result in $2^{\circ}$. Finally, routine arguments show that the results in $1^{\circ}-3^{\circ}$ hold for an arbitrary measurable space $(X, Q)$, since they hold for a separable metric space (see [7]).

\section{REFERENCES}

1. D. Blackwell and C. Ryll-Nardzewski, Non-existence of every'here proper conditional distributions, Ann. Math. Statist. 34 (1963), 223-225.

2. J. P. Burgess, Careful choices: A last word on Borel selectors, preprint, 1979.

3. K. Kuratowski, Topologv, vol. I (5th ed.), Academic Press, New York, 1966.

4. A. Maitra, An effective selection theorem, J. Symbolic Logic 47 (1982), 388-394.

5. Y. N. Moschovakis, Descriptive set theon, North-Holland, Amsterdam, 1980.

6. H. Sarbadhikari, Some uniformization results, Fund. Math. 97 (1977), 209-214.

7. V. V. Srivatsa, Measurable sets in product spaces and their parametrizations, doctoral dissertation. Indian Statistical Institute, Calcutta, 1981.

8. S. M. Srivastava, Selection theorems for $G_{\delta}$-valued multifunctions, Trans. Amer. Math. Soc. 254 (1979), 283-293.

9. R. L. Vaught, Invariant sets in logic and topologl, Fund. Math. 82 (1974), 269-294.

Department of Statistics, University of California, Berkeley, California 94720

Indian Statistical Institute, Calcutta, India 